\title{
ON THE ABSOLUTE RIESZ SUMMABILITY OF FOURIER SERIÉS AND ITS CON JUGATE SERIES
}

\author{
BY \\ T. PATI
}

1.1. Definition. Let $\sum_{1}^{\infty} a_{n}$ be a given infinite series, and $\lambda_{n}$ a positive, steadily increasing, monotonic function of $n$, tending to infinity with $n$. We write

$$
A_{\lambda}(\omega)=A_{\lambda}^{0}(\omega)=\sum_{\lambda_{n} \leqq \omega} a_{n}
$$

and

$$
A_{\lambda}^{r}(\omega)=\sum_{\lambda_{n} \leqq \omega}\left(\omega-\lambda_{n}\right)^{r} a_{n} .
$$

The series $\sum_{1}^{\infty} a_{n}$ is said to be absolutely summable $(R, \lambda, r)$, or simply summable $|R, \lambda, r|, r \geqq 0$, if $A_{\lambda}^{r}(\omega) / \omega^{r}$ is of bounded variation in $(A, \infty)$, where $A$ is a finite positive number $[6 ; 7]\left({ }^{1}\right)$. We may, for example, take $A=\lambda_{1}$.

The above definition can also be put in the following equivalent form by defining $\lambda$ suitably at nonintegral points and by a change of variable.

Alternative Definition. Let $\lambda=\lambda(\omega)$ be a continuous, differentiable, and monotonic increasing function of $\omega$ in $(K, \infty), K$ being a positive constant, and let it tend to infinity with $\omega$. Suppose that $\sum_{1}^{\infty} a_{n}$ is a given infinite series, and write

$$
c_{r}(\omega)=\sum_{n \leqq \omega}\{\lambda(\omega)-\lambda(n)\}^{r} a_{n} \quad(r \geqq 0) .
$$

Then the series $\sum_{1}^{\infty} a_{n}$ is summable $|R, \lambda, r|, r \geqq 0$, if the integral

$$
\int_{A}^{\infty}\left|d\left[c_{r}(\omega) /\{\lambda(\omega)\} r\right]\right|
$$

where $A$ is a finite positive number, is convergent. Now, for $r>0, m<\omega$ $<m+1$,

$$
\frac{d}{d \omega}\left[c_{r}(\omega) /\{\lambda(\omega)\}^{r}\right]=\frac{r \lambda^{\prime}(\omega)}{\{\lambda(\omega)\}^{r+1}} \sum_{n \leqq \omega}\{\lambda(\omega)-\lambda(n)\}^{r-1} \lambda(n) a_{n} .
$$

Hence $\sum_{1}^{\infty} a_{n}$ is summable $|R, \lambda, r|, r>0$, if

Received by the editors December 5, 1952.

(1) The numbers in brackets refer to the bibliography. 


$$
\int_{A}^{\infty}\left|\frac{r \lambda^{\prime}(\omega)}{\{\lambda(\omega)\}^{r+1}} \sum_{n \leqq \omega}\{\lambda(\omega)-\lambda(n)\}^{r-1} \lambda(n) a_{n}\right| d \omega<\infty .
$$

It is evident that summability $|R, \lambda, 0|$ is equivalent to absolute convergence.

For convenience we shall adopt the alternative definition throughout the present paper.

1.2. Let $f(t)$ be a periodic function with period $2 \pi$ and integrable $(L)$ over $(-\pi, \pi)$. Without loss of generality the constant term in the Fourier series of $f(t)$ can be taken to be zero, so that

$$
f(t) \sim \sum_{1}^{\infty}\left(a_{n} \cos n t+b_{n} \sin n t\right)=\sum_{1}^{\infty} A_{n}(t)
$$

and

$$
\int_{-\pi}^{\pi} f(t) d t=0
$$

Then the conjugate series of the Fourier series of $f(t)$ is given by

$$
\sum_{1}^{\infty}\left(b_{n} \cos n t-a_{n} \sin n t\right)=\sum_{1}^{\infty} B_{n}(t) .
$$

Throughout the paper we use the following notations:

$$
\begin{array}{rlr}
\phi(t) & =\{f(x+t)+f(x-t)\} / 2 ; & \\
\psi(t) & =\{f(x+t)-f(x-t)\} / 2 ; & \\
P(t) & =\sum_{0}^{r-1}\left(\theta_{i} t^{i} / i !\right), & \text { where the } \theta^{\prime} \text { s are arbitrary; } \\
g(t) & =\left[\{f(x+t)-P(t)\}+(-1)^{r}\{f(x-t)-P(-t)\}\right] / 2 ; & \\
h(t) & =\left[\{f(x+t)-P(t)\}-(-1)^{r}\{f(x-t)-P(-t)\}\right] / 2 ; & \\
\Phi_{\sigma}(t) & =\frac{1}{\Gamma(\sigma)} \int_{0}^{t}(t-u)^{\sigma-1} \phi(u) d u & (\sigma>0) ; \\
\Phi_{0}(t) & =\phi(t) ; & (\sigma \geqq 0) ; \\
\phi_{\sigma}(t) & =\Gamma(\sigma+1) t^{-\sigma} \Phi_{\sigma}(t)
\end{array}
$$

$\Psi_{\sigma}(t), \psi_{\sigma}(t), G_{\sigma}(t), g_{\sigma}(t), H_{\sigma}(t)$, and $h_{\sigma}(t)$ have similar meanings;

$$
\begin{aligned}
\gamma_{\alpha, r}(t) & =g_{\alpha-r}(t) / t^{r} ; \quad \theta_{\alpha, r}(t)=h_{\alpha-r}(t) / t^{r} ; \\
e(\omega) & =\exp \left\{(\log \omega)^{1+1 / \alpha}\right\} ; \\
E(\omega, t) & =\sum_{n \leqq \omega}\{e(\omega)-e(n)\}^{\alpha} e(n) \cos n t ; \quad E^{(r)}(\omega, t)=\frac{\partial^{r}}{\partial t^{r}} E(\omega, t) ;
\end{aligned}
$$




$$
\begin{gathered}
\bar{E}(\omega, t)=\sum_{n \leqq \omega}\{e(\omega)-e(n)\} \alpha e(n) \sin n t ; \quad \bar{E}^{(r)}(\omega, t)=\frac{\partial^{r}}{\partial t^{r}} \bar{E}(\omega, t) \\
g(\omega, t)=\int_{0}^{t} \frac{u^{\alpha}}{\log (k / u)} E^{(\alpha)}(\omega, u) d u ; \quad h(\omega, t)=\int_{t}^{\pi} \frac{u^{\alpha}}{\log (k / u)} E^{(\alpha)}(\omega, u) d u \\
(F(t))_{r}=\frac{\partial^{r}}{\partial t^{r}} F(t) .
\end{gathered}
$$

1.3. The object of this paper is to generalise our previous work $\left({ }^{2}\right)$ done in the subject and to establish theorems of a very general character concerning the absolute Riesz summability, for a rapidly increasing type, of the Fourier series, its conjugate series, and their derived series. These theorems are stated in $\$ 2.1$. It will be seen that Theorem 1 is the analogue, for absolute summability, of a theorem on ordinary Riesz summability of Fourier series, recently obtained by Wang [9], for the case in which $\alpha$ is a positive integer $\geqq 1$. It may be mentioned that the four theorems of this paper include as particular cases Theorems 4, 6, 7, and 8 respectively, recently published [5] by Mohanty in the Proceedings of the London Mathematical Society.

I take this opportunity of expressing my deep indebtedness to Dr. B. N. Prasad for his kind encouragement and valuable suggestions during the preparation of this paper.

2.1. We establish the following theorems.

TheOREM 1. If $\alpha$ is an integer $\geqq 1, \operatorname{and}\left({ }^{3}\right) \phi_{\alpha}(t) \log (k / t)$ is of bounded variation in $(0, \pi)$, then the Fourier series of $f(t)$, at $t=x$, is summable $\mid R$, $e(\omega), \alpha+1 \mid$.

Theorem 2. If $\alpha$ is an integer $\geqq 1$, and if (i) $\psi_{\alpha}(t) \log (k / t)$ is of bounded variation in $(0, \pi)$ and (ii) $\left|\psi_{\alpha}(t)\right| / t$ is integrable $(L)$ over $(0, \pi)$, then the conjugate series of the Fourier series of $f(t)$, at $t=x$, is summable $|R, e(\omega), \alpha+1|$.

THEOREM 3. If $r$ is an integer $\geqq 1$, and $\gamma_{\alpha, r}(t) \log (k / t)$ is of bounded variation in $(0, \pi)$, then the rth derived series of the Fourier series of $f(t)$, at $t=x$, is summable $|R, e(\omega), \alpha+1|$, for every integral $\alpha \geqq r$.

THEOREM 4. If $r$ is an integer $\geqq 1$, and if (i) $\theta_{\alpha, r}(t) \log (k / t)$ is of bounded variation in $(0, \pi)$ and (ii) $\left|\theta_{\alpha, r}(t)\right| / t$ is integrable $(L)$ over $(0, \pi)$, then the rth derived series of the conjugate series of the Fourier series of $f(t)$, at $t=x$, is summable $|R, e(\omega), \alpha+1|$, for every integral $\alpha \geqq r$.

2.2. We require a number of lemmas for the proofs of our theorems.

(2) Under publication in the Bulletin of the Calcutta Mathematical Society.

(3) In the enunciation of the theorems of this paper it is sufficient to take $k>\pi$. Since it is immaterial what particular value $k$ has, for the sake of convenience we assume $k>e^{\alpha+2} \pi$ in the proofs of Theorems 1 and 3. 
Lemma $1[6,7]$. If a series $\sum_{1}^{\infty} a_{n}$ is summable $|R, \lambda, r|, r \geqq 0$, then it is summable $\left|R, \lambda, r^{\prime}\right|$ for $r^{\prime}>r$.

Lemma 2. The Fourier series of the special functions

$$
\left(\log \left|\frac{k}{t}\right|\right)^{-1},\left(\log \left|\frac{k}{t}\right|\right)^{-2}, \cdots,\left(\log \left|\frac{k}{t}\right|\right)^{-\alpha-1}
$$

are all absolutely convergent at $t=0$.

The absolute convergence, at $t=0$, of the Fourier series of the special function $(\log |k / t|)^{-1}$ has been proved [4] by Mohanty. The proofs of the absolute convergence, at $t=0$, of the Fourier series of all the other special functions proceed on similar lines.

Lemma 3. Let $C_{n}^{(k)}, S_{n}^{(k)}$, and $\bar{S}_{n}^{(k)}$ denote the nth Cesdro-sums of order $k$ $(k \geqq 0)$ corresponding to the series

$$
\sum_{1}^{\infty}(-1)^{n} n^{\rho}, \quad \sum_{1}^{\infty}(\cos n t)_{\rho}, \quad \text { and } \quad \sum_{1}^{\infty}(\sin n t)_{\rho}
$$

respectively. Then

$$
\begin{array}{ll}
S_{n}^{(k)}=O\left(n^{\rho+k+1}\right) & \text { for } 0<t \leqq 1 / n ; \\
S_{n}^{(k)}=O\left(n^{\rho} t^{-(k+1)}\right)+O\left(n^{k-1} t^{-(\rho+2)}\right) & \text { for } 1 / n<t \leqq \pi ; \\
\bar{S}_{n}^{(k)}=O\left(n^{\rho+k+1}\right) & \text { for } 0<t \leqq 1 / n ; \\
\bar{S}_{n}^{(k)}=O\left(n^{\rho} t^{-(k+1)}\right)+O\left(n^{k} t^{-(\rho+1)}\right) & \text { for } 1 / n<t \leqq \pi ;
\end{array}
$$

(v) when $\rho$ is an even integer $\geqq 2$,

$$
C_{n}^{(k)}=O\left(n^{\max (\rho, k-1)}\right) .
$$

Proof. We write

$$
1 / 2+\cos t+\cos 2 t+\cdots=c_{0}+c_{1}+c_{2}+\cdots=\sum c_{n} .
$$

Let $s_{n}^{(k)}$ denote the $n$th Cesàro-sum of order $k$ corresponding to the series $\sum c_{n}$. We first estimate $s_{n}^{(k)}$ for $0<t \leqq 1 / n$ and $1 / n<t \leqq \pi$. If $0<t \leqq 1 / n$, then $c_{n}=O(1), s_{n}=O(n)$, and thus $s_{n}^{(k)}=O\left(n^{k+1}\right)$ uniformly in $0<t \leqq 1 / n$. When $1 / n$ $<t \leqq \pi$, proceeding as in Hardy's Divergent series [2, p. 361], we get

$$
s_{n}^{(k)}=\Omega(n)+W(n),
$$

where

$$
\Omega(n)=\sin \{(n+k / 2+1 / 2) t-k \pi / 2\} /(2 \sin t / 2)^{k+1},
$$

and 


$$
W(n)=\frac{1}{4 \pi i} \int_{C} \frac{1-u^{2}}{1-2 u \cos t+u^{2}} \cdot \frac{d u}{(1-u)^{k+1} u^{n+1}},
$$

$C$ being the lacet formed by the circle $|u-1|=\tau\left(\tau<\left|1-e^{i t}\right|\right)$, and the line $(1+\tau, \infty)$ described twice in opposite directions. It is plain that

$$
\Omega(n)=O\left(t^{-k-1}\right)
$$

uniformly. Also, taking $\tau=1 / 2 n$, we estimate that

$$
W(n)=O\left(n^{k-1} t^{-2}\right) .
$$

Hence, we finally have

$$
s_{n}^{(k)}=O\left(t^{-k-1}\right)+O\left(n^{k-1} t^{-2}\right) \quad \text { for } 1 / n<t \leqq \pi .
$$

We now proceed to derive the results (i) and (ii) of Lemma 3. Since $S_{n}^{(\mathbf{k})}$ is derived from $\sum(\cos n t)_{p}$ in the same manner as $s_{n}^{(k)}$ is derived from $\sum c_{n}$, we have

$$
S_{n}^{(k)}=O\left(n^{k+\rho+1}\right)
$$

uniformly in $0<t \leqq 1 / n$. When $1 / n<t \leqq \pi$,

$$
S_{n}^{(k)}=\Omega^{(\rho)}(n)+W^{(\rho)}(n),
$$

where $\Omega^{(\rho)}(n)$ and $W^{(\rho)}(n)$ are the $\rho$ th derivatives with respect to $t$ of $\Omega(n)$ and $W(n)$ respectively. Using Leibnitz's formula for the derivative of a product and observing that

$$
(\sin \{(n+k / 2+1 / 2) t-k \pi / 2\})_{\rho-\lambda}=O\left(n^{\rho-\lambda}\right),
$$

while

$$
\left(1 /(2 \sin t / 2)^{k+1}\right)_{\lambda}=O\left(t^{-k-\lambda-1}\right),
$$

we infer that

$$
\Omega^{(\rho)}(n)=O\left(n^{\rho} t^{(k+1)}\right) .
$$

Next, treating $W^{(\rho)}(n)$ in just the same way as we treated $W(n)$, we find that

$$
W^{(\rho)}(n)=O\left(n^{k-1} t^{(\rho+2)}\right) .
$$

Thus finally

$$
S_{n}^{(k)}=O\left(n^{p} t^{-(k+1)}\right)+O\left(n^{k-1} t^{-(\rho+2)}\right) \quad \text { for } 1 / n<t \leqq \pi .
$$

The results (iii) and (iv) of the lemma follow from the results (i) and (ii), when we observe that $(\sin n t)_{p}=n(\cos n t)_{p-1}$. The result $(\mathrm{v})$ follows from the result (ii) by using the identity $\cos n \pi=(-1)^{n}$.

Lemma 4 [3]. Let 


$$
A_{\lambda}(x)=A_{\lambda}^{0}(x)=\sum_{\lambda_{n} \leqq x} a_{n}
$$

and

$$
A_{\lambda}^{r}(x)=\sum_{\lambda_{n} \leqq x}\left(x-\lambda_{n}\right)^{r} a_{n}
$$

Then, if $k$ is a positive integer,

$$
A_{\lambda}(x)=\frac{1}{k !}\left(\frac{d}{d x}\right)^{k} A_{\lambda}^{k}(x) .
$$

LEMMA 5. The nth derivative of $\{F(x)\}^{m}$ is the sum of a number of terms of the form

$$
K\{F(x)\}^{m-r}\left\{F^{(1)}(x)\right\} \alpha_{1}\left\{F^{(2)}(x)\right\}^{\alpha_{2}} \ldots\left\{F^{(n)}(x)\right\} \alpha_{n},
$$

where the $K$ 's are constants, $r \leqq n$, and the $\alpha$ 's are positive integers or zeros such that

$$
\sum_{1}^{n} \alpha_{\nu}=r ; \quad \sum_{1}^{n} \nu \alpha_{\nu}=n
$$

Further, if $m$ is a positive integer, then $r \leqq m$.

This is a particular case of a result, due to Faa di Bruno [8, p. 89], on the $n$th derivative of a function of a function.

LEMMA 6. If $\rho$ is an even integer such that $2 \leqq \rho \leqq \alpha-1$, then the integral

$$
\int_{1}^{\infty} \frac{(\log \omega)^{1 / \alpha}}{\omega e^{\alpha+1}(\omega)}\left|E^{(\rho)}(\omega, \pi)\right| d \omega
$$

is convergent.

Proof. We have

$$
E^{(\rho)}(\omega, \pi)=(-1)^{\rho / 2} \sum_{n \leqq \omega}\{e(\omega)-e(n)\}^{\alpha} e(n)(-1)^{n} n^{\rho} .
$$

We first prove that

$$
\sum_{n \leqq \omega}\{e(\omega)-e(n)\}^{\alpha} e(n)(-1)^{n} n^{\rho}=O\left\{\frac{\log \omega}{\omega} e^{\alpha+1}(\omega)\right\} .
$$

Evidently it suffices for our purpose to show that

$$
e(\omega) \sum_{n \leq \omega}\{e(\omega)-e(n)\}^{\alpha}(-1)^{n} n^{\rho}=O\left\{\frac{\log \omega}{\omega} e^{\alpha+1}(\omega)\right\}
$$

and 


$$
\sum_{n \leqq \omega}\{e(\omega)-e(n)\}^{\alpha+1}(-1)^{n} n^{\rho}=O\left\{\frac{\log \omega}{\omega} e^{\alpha+1}(\omega)\right\} .
$$

We use the following notations:

$$
\mathfrak{S}(x)=\sum_{n \leqq x}(-1)^{n} n^{\rho} ; \complement^{\kappa}(x)=\sum_{n \leqq x}(x-n)^{\kappa}(-1)^{n} n^{\rho} \quad(\kappa=1,2, \cdots) .
$$

We observe that

$$
\mathfrak{S}^{\alpha}(x)=O\left(x^{\alpha-1}\right) .
$$

To prove this suppose $m \leqq x<m+1$. Then

$$
{ }^{(}{ }^{\alpha}(x)=\sum_{1}^{m}(x-n)^{\alpha}(-1)^{n} n^{\rho}=\sum_{1}^{m-1} \Delta(x-n){ }^{\alpha} C_{n}^{(0)}+(x-m){ }^{\alpha} C_{m}^{(0)} \text {, }
$$

following the notations of Lemma 3. Again

$$
\sum_{1}^{m-1} \Delta(x-n)^{\alpha} C_{n}^{(0)}=\sum_{1}^{m-2} \Delta^{2}(x-n)^{\alpha} C_{n}^{(1)}+\left[\Delta(x-n)^{\alpha}\right]_{n=m-1} C_{m-1}^{(1)}
$$

Repeating Abel's transformation, and applying Lemma 3, we easily get the inequality (2.24). Now

$$
\begin{aligned}
\sum_{n \leqq \omega}\{e(\omega)-e(n)\}^{\alpha}(-1)^{n} n^{\rho} & =-\int_{1}^{\omega} \Subset(x) \frac{d}{d x}\{e(\omega)-e(x)\}^{\alpha} d x \\
& =-\left(\int_{1}^{e}+\int_{0}^{\omega}\right) \Subset(x) \frac{d}{d x}\{e(\omega)-e(x)\}^{\alpha} d x \\
& =-\left(I_{1}+I_{2}\right), \text { say. }
\end{aligned}
$$

Evidently

$$
e(\omega) I_{1}=O\left((\log \omega / \omega) e^{\alpha+1}(\omega)\right) .
$$

Next

$$
\begin{aligned}
I_{2} & =\int_{0}^{\omega} \mathfrak{S}(x) \frac{d}{d x}\{e(\omega)-e(x)\}^{\alpha} d x \\
& =\frac{1}{\alpha !} \int_{e}^{\omega}\left(\frac{d}{d x}\right)^{\alpha} \mathfrak{S} \alpha(x) \frac{d}{d x}\{e(\omega)-e(x)\} \alpha d x,
\end{aligned}
$$

by Lemma 4 . Integrating the last integral by parts, we have

$$
\begin{aligned}
& I_{2}=O\left(\left|\mathfrak{S}^{\alpha}(\omega)\right|\left\{e^{(1)}(\omega)\right\}^{\alpha}\right)+O\left(e^{\alpha-1}(\omega)\right) \\
& +O\left(\left|\int_{e}^{\omega} \Im^{\alpha}(x)\left(\frac{d}{d x}\right)^{\alpha+1}\{e(\omega)-e(x)\}^{\alpha} d x\right|\right) \text {. }
\end{aligned}
$$


Now, applying Lemma 5 and the binomial theorem, we see that

$$
\mathfrak{I}=\int_{0}^{\omega} \mathfrak{E}^{\alpha}(x)\left(\frac{d}{d x}\right)^{\alpha+1}\{e(\omega)-e(x)\}^{\alpha} d x
$$

is a sum of constant multiples of terms of the type

$$
e^{\alpha-r}(\omega) \int_{0}^{\omega} \mathfrak{S}^{\alpha}(x) e^{\beta}(x)\left\{e^{(1)}(x)\right\}^{\beta_{1}} \cdots\left\{e^{(\alpha+1)}(x)\right\}^{\beta_{\alpha+1}} d x,
$$

where the $\beta$ 's are positive integers or zeros such that

$$
\begin{gathered}
0<\beta+\beta_{1}+\cdots+\beta_{\alpha+1}=r<\alpha, \\
\beta_{1}+2 \beta_{2}+3 \beta_{3}+\cdots+(\alpha+1) \beta_{\alpha+1}=\alpha+1 .
\end{gathered}
$$

Now, using (2.24) and the inequalities

$$
\begin{aligned}
e^{(1)}(x) & =o\left\{\frac{(\log x)^{1 / \alpha}}{x} e(x)\right\}, \\
e^{(2)}(x) & =o\left\{\frac{(\log x)^{2 / \alpha}}{x^{2}} e(x)\right\}, \\
\ldots \ldots & \ldots \ldots \\
e^{(\alpha+1)}(x) & =o\left\{\frac{(\log x)^{(\alpha+1) / \alpha}}{x^{\alpha+1}} e(x)\right\},
\end{aligned}
$$

we observe that

$$
\begin{aligned}
\int_{0}^{\omega}{ }^{\alpha}(x) e^{\beta}(x)\left\{e^{(1)}(x)\right\}^{\beta_{1}} \cdots\left\{e^{(\alpha+1)}(x)\right\}^{\beta_{\alpha+1}} d x & \\
& =O\left(\int_{0}^{\omega} x^{\alpha-1} e^{r}(x)\left\{\frac{(\log x)^{1 / \alpha}}{x}\right\}^{\alpha+1} d x\right) \\
& =O\left(\int_{0}^{\omega} \frac{(\log x)^{1+1 / \alpha}}{x^{2}} e^{r}(x) d x\right) .
\end{aligned}
$$

Now, if $r=1$,

$$
\begin{aligned}
\int_{0}^{\omega} \frac{(\log x)^{1+1 / \alpha}}{x^{2}} e^{r}(x) d x & =O\left(\int_{0}^{\omega} \frac{\log x}{x} e^{(1)}(x) d x\right) \\
& =O\left(\left[\frac{\log x}{x} e(x)\right]_{0}^{\omega}+\int_{0}^{\omega} e(x) \frac{\log x-1}{x^{2}} d x\right) \\
& =O\left(\frac{\log \omega}{\omega} e(\omega)\right) .
\end{aligned}
$$


Again, if $r=2,3, \cdots$,

$$
\begin{aligned}
\int_{e}^{\omega} \frac{(\log x)^{1+1 / \alpha}}{x^{2}} e^{r}(x) d x & =O\left(\int_{e}^{\omega} e^{r-1}(x) \frac{\log x}{x} e^{(1)}(x) d x\right) \\
& =O\left(\frac{\log \omega}{\omega} e^{r-1}(\omega) e(\omega)\right)=O\left(\frac{\log \omega}{\omega} e^{r}(\omega)\right) .
\end{aligned}
$$

Hence $J$ is a sum of constant multiples of terms each of which equals $O\{(\log \omega / \omega)\}$, so that, finally, $e(\omega) I_{2}=O\left\{(\log \omega / \omega) e^{\alpha+1}(\omega)\right\}$.

The proof of (2.23) proceeds on essentially the same lines as that of (2.22); the integral

$$
\int_{e}^{\omega}\left(\varsigma^{\alpha}(x)\left(\frac{d}{d x}\right)^{\alpha+1}\{e(\omega)-e(x)\}^{\alpha+1} d x\right.
$$

replaces $J$ with corresponding differences in details. This completes the proof of (2.21). Hence

$$
\int_{1}^{\infty} \frac{(\log \omega)^{1 / \alpha}}{\omega e^{\alpha+1}(\omega)}\left|E^{(\rho)}(\omega, \pi)\right| d \omega=O\left(\int_{1}^{\infty} \frac{(\log \omega)^{1+1 / \alpha}}{\omega^{2}} d \omega\right)=O(1) .
$$

This completes the proof of Lemma 6.

LEMMA 7. If $\rho$ is zero or a positive integer $\leqq \alpha-1$, then

$$
\sum_{n \leqq \omega}\{e(\omega)-e(n)\}^{\alpha} e(n) n^{\rho}=O\left\{\omega^{\rho+1} e^{\alpha+1}(\omega) /(\log \omega)^{1 / \alpha}\right\} .
$$

Proof. For $m \leqq \omega<m+1$,

$$
\begin{aligned}
\sum_{n \leqq \omega}\{e(\omega)-e(n)\} \alpha & e(n) n^{\rho}=\sum_{1}^{m}\{e(\omega)-e(n)\} \alpha e(n) n^{\rho} \\
= & \sum_{1}^{m-1} \Delta\{e(\omega)-e(n)\}^{\alpha} \sum_{1}^{n} e(\nu) \nu^{\rho}+\{e(\omega)-e(m)\} \alpha \sum_{1}^{m} e(\nu) \nu^{\rho} \\
= & O\left[\sum_{1}^{m-1}\{e(n+1)-e(n)\}\{e(\omega)-e(n)\}^{\alpha-1} \sum_{1}^{n} e(\nu) \nu^{\rho}\right] \\
& +\{e(\omega)-e(m)\} \alpha \sum_{1}^{m} e(\nu) \nu^{\rho} \\
= & O\left[e^{\alpha-1}(\omega) \sum_{1}^{m-1} \frac{\{\log (n+1)\}^{1 / \alpha}}{n+1} e(n+1) \frac{n^{\rho} n e(n)}{(\log n)^{1 / \alpha}}\right] \\
& +O\left\{\frac{\log \omega}{\omega^{\alpha}} e^{\alpha}(\omega) \frac{\omega^{\rho} \omega e(\omega)}{(\log \omega)^{1 / \alpha}}\right\}
\end{aligned}
$$

since $e(n+1)-e(n)=O\left[\{\log (n+1)\}^{1 / \alpha} e(n+1) /(n+1)\right]$ and 


$$
\sum_{1}^{n} e(\nu)=O\left\{n e(n) /(\log n)^{1 / \alpha}\right\}
$$

Thus

$$
\begin{aligned}
\sum_{n \leqq \omega}\{e(\omega)-e(n)\} \alpha e(n) n^{\rho} & \\
= & O\left\{e^{\alpha-1}(\omega) \sum_{1}^{m-1} e(n+1) n^{\rho} e(n)\right\}+O\left\{(\log \omega)^{1-1 / \alpha} e^{\alpha+1}(\omega) / \omega^{\alpha-1-\rho}\right\} \\
& =O\left\{e^{\alpha+1}(\omega) \omega^{\rho+1} /(\log \omega)^{1 / \alpha}\right\} .
\end{aligned}
$$

This completes the proof of Lemma 7.

Lemma 8.

$$
E^{(\alpha-1)}(\omega, t)=O\left\{(\log \omega / \omega) e^{\alpha+1}(\omega) t^{-(\alpha+1)}\right\} .
$$

Proof. The proof of this lemma proceeds along the same lines as that of Lemma 6. Suffice it to observe that in the analysis $\mathfrak{\complement}(x)$ and $\mathfrak{\complement}{ }^{\alpha}(x)$ will be replaced by $\mathfrak{S}(x)$ and $\mathfrak{S}^{\alpha}(x)$, where

$$
\mathfrak{S}(x)=\sum_{n \leqq x}(\cos n t)_{\alpha-1} \text { and } \Im^{\alpha}(x)=\sum_{n \leqq x}(x-n)^{\alpha}(\cos n t)_{\alpha-1} .
$$

Also, by repeated application of Abel's transformation and Lemma 3, as in the proof of (2.24), we estimate that $\mathfrak{S}^{\alpha}(x)=O\left(x^{\alpha-1} t^{-(\alpha+1)}\right)$.

Lemma 9. If $\rho$ is zero or a positive integer $\leqq \alpha-1$, then

$$
\bar{E}^{(\rho)}(\omega, t)=O\left\{(\log \omega / \omega) e^{\alpha+1}(\omega) t^{-(\rho+2)}\right\} .
$$

\section{Proof.}

$$
\begin{aligned}
\bar{E}^{(\rho)}(\omega, t)= & \sum_{n \leqq \omega}\{e(\omega)-e(n)\}^{\alpha} e(n)(\sin n t)_{\rho} \\
= & -\int_{1}^{\omega}\left\{\sum_{n \leqq x}(\sin n t)_{\rho}\right\} \frac{d}{d x}\left[\{e(\omega)-e(x)\}^{\alpha} e(x)\right] d x \\
= & -\frac{1}{2}\left(\cot \frac{1}{2} t\right)_{\rho} \int_{1}^{\omega} \frac{d}{d x}\left[\{e(\omega)-e(x)\}^{\alpha} e(x)\right] d x \\
& -\frac{1}{2} \int_{1}^{\omega}(\sin [x] t)_{\rho} \frac{d}{d x}\left[\{e(\omega)-e(x)\}^{\alpha} e(x)\right] d x \\
& +\frac{1}{2} \int_{1}^{\omega}\left(\cos [x] t \cot \frac{1}{2} t\right)_{\rho} \frac{d}{d x}\left[\{e(\omega)-e(x)\}^{\alpha} e(x)\right] d x \\
= & -\frac{1}{2}\left(\cot \frac{1}{2} t\right)_{\rho} I_{1}-\frac{1}{2} I_{2}+\frac{1}{2} I_{3}, \text { say. }
\end{aligned}
$$


Evidently

$$
\frac{1}{2}\left(\cot \frac{1}{2} t\right)_{\rho} I_{1}=O\left\{\frac{\log \omega}{\omega} e^{\alpha+1}(\omega) t^{-(\rho+1)}\right\} .
$$

For estimating $I_{2}$ it suffices to consider only

$$
e(\omega) \int_{1}^{\omega}(\sin [x] t)_{p} \frac{d}{d x}\{e(\omega)-e(x)\}^{\alpha} d x
$$

and

$$
\int_{1}^{\omega}(\sin [x] t)_{\rho} \frac{d}{d x}\{e(\omega)-e(x)\}^{\alpha+1} d x
$$

Clearly

$$
\begin{aligned}
e(\omega) \int_{1}^{\omega}(\sin [x] t)_{\rho} \frac{d}{d x}\{e(\omega)-e(x)\}^{\alpha} d x \\
=O\left\{\frac{\log \omega}{\omega} e^{\alpha+1}(\omega)\right\}+e(\omega) \int_{0}^{\omega}(\sin [x] t)_{\rho} \frac{d}{d x}\{e(\omega)-e(x)\}^{\alpha} d x,
\end{aligned}
$$

and

$$
\begin{aligned}
& \int_{e}^{\omega}(\sin [x] t)_{\rho} \frac{d}{d x}\{e(\omega)-e(x)\}^{\alpha} d x \\
&=\frac{1}{\alpha !} \int_{0}^{\omega}\left(\frac{d}{d x}\right)^{\alpha} \widetilde{\Xi}^{\alpha}(x) \frac{d}{d x}\{e(\omega)-e(x)\}^{\alpha} d x,
\end{aligned}
$$

where

$$
\tilde{\Phi}^{\alpha}(x)=\sum_{1}^{[x]-1} \Delta(x-n)^{\alpha}(\sin n t)_{\rho}+(x-[x])^{\alpha}(\sin [x] t)_{\rho}=O\left(x^{\alpha-1} t^{-(\rho+1)}\right),
$$

by repeated application of Abel's transformation and Lemma 3, as in the proof of (2.24). Proceeding with the last integral as in the proof of Lemma 6, we have

$$
e(\omega) \int_{1}^{\omega}(\sin [x] t)_{\rho} \frac{d}{d x}\{e(\omega)-e(x)\}^{\alpha} d x=O\left\{\frac{\log \omega}{\omega} e^{\alpha+1}(\omega) t^{-(\rho+1)}\right\} .
$$

Similarly

$$
\int_{1}^{\omega}(\sin [x] t)_{\rho} \frac{d}{d x}\{e(\omega)-e(x)\}^{\alpha+1} d x=O\left\{\frac{\log \omega}{\omega} e^{\alpha+1}(\omega) t^{-(\rho+1)}\right\} .
$$

Thus, finally, 


$$
I_{2}=O\left\{\frac{\log \omega}{\omega} e^{\alpha+1}(\omega) t^{-(\rho+1)}\right\} .
$$

Also, by parallel reasoning, for $r=0,1, \cdots, \rho$,

$$
\int_{1}^{\omega}(\cos [x] t)_{\rho-r} \frac{d}{d x}\left[\{e(\omega)-e(x)\}^{\alpha} e(x)\right] d x=O\left\{\frac{\log \omega}{\omega} e^{\alpha+1}(\omega) t^{-(\rho-r+1)}\right\} .
$$

Now, since $(\cot t / 2)_{r}=O\left(t^{-(r+1)}\right)$,

$$
I_{3}=O\left\{\frac{\log \omega}{\omega} e^{\alpha+1}(\omega) t^{-(\rho+2)}\right\} .
$$

Combining the estimates (2.25), (2.26), and (2.27), the lemma is proved.

Lemma 10. If $\rho$ is an odd integer such that $1 \leqq \rho \leqq \alpha-1$, then the integral

$$
\int_{1}^{\infty} \frac{(\log \omega)^{1 / \alpha}}{\omega e^{\alpha+1}(\omega)}\left|\bar{E}^{(\rho)}(\omega, \pi)\right| d \omega
$$

is convergent.

The result follows immediately on application of Lemma 9.

LEMMA 11. If $\rho$ is an integer such that $1 \leqq \rho \leqq \alpha-1$, then the series $\sum(-1)^{n} n^{\rho}$ is summable $|R, e(\omega), \alpha+1|$.

This is essentially a combination of the results of Lemma 6 and Lemma 10.

LEMMA 12 [1]. If the series $\sum_{1}^{\infty} a_{n}$ is summable $|R, \lambda, r|, r>0$, and $\mu$ is a logarithmico-exponential function of $\lambda$ such that $\mu=O\left(\lambda^{\Delta}\right)$, where $\Delta$ is a constant, then the series $\sum_{1}^{\infty} a_{n}$ is summable $|R, \mu, r|$.

- LemMa 13 [5]. The necessary and sufficient conditions that (i) $F(t) \log (k / t)$ be of bounded variation in $(0, \eta)$ and (ii) $|F(t)| / t$ be integrable $(L)$ over $(0, \eta)$, $\eta$ being positive, are that $\int_{0}^{\eta} \log (k / t)|d F(t)|<\infty$ and $F(+0)=0$.

Lemma 14 [5]. If $F(+0)=0$ and $\int_{0}^{x} \log (k / t)|d F(t)|<\infty$, then the series $\sum v_{n}$, where

$$
v_{n}=\int_{0}^{\pi} F(t) \sin n t d t=-F(\pi) \frac{\cos n \pi}{n}+\int_{0}^{\pi} \frac{\cos n t}{n} d F(t),
$$

is summable $\left|R, \exp \left(\omega^{\delta}\right), 1\right|$, where $0<\delta<1$.

3.1. Proof of Theorem 1. Since

$$
A_{n}(x)=\frac{2}{\pi} \int_{0}^{\pi} \phi(t) \cos n t d t,
$$

we have to show that, under the hypotheses of the theorem, the integral 


$$
\int_{1}^{\infty} \frac{(\log \omega)^{1 / \alpha}}{\omega e^{\alpha+1}(\omega)}\left|\int_{0}^{\pi} \phi(t) E(\omega, t) d t\right| d \omega
$$

is convergent. Integrating by parts $\alpha$ times,

$$
\begin{aligned}
\int_{0}^{\pi} \phi(t) E(\omega, t) d t= & {\left[\sum_{1}^{\alpha}(-1)^{\rho-1} \Phi_{\rho}(t) E^{(\rho-1)}(\omega, t)\right]_{0}^{\pi} } \\
& +(-1)^{\alpha} \int_{0}^{\pi} \Phi_{\alpha}(t) E^{(\alpha)}(\omega, t) d t .
\end{aligned}
$$

Also

$$
\begin{aligned}
\int_{0}^{\pi} \Phi_{\alpha}(t) E^{(\alpha)}(\omega, t) d t= & \frac{1}{\Gamma(\alpha+1)} \int_{0}^{\pi} t^{\alpha} \phi_{\alpha}(t) E^{(\alpha)}(\omega, t) d t \\
& =\frac{1}{\Gamma(\alpha+1)} \int_{0}^{\pi} \phi_{\alpha}(t) \log (k / t) \frac{t^{\alpha}}{\log (k / t)} E^{(\alpha)}(\omega, t) d t \\
& =\frac{1}{\Gamma(\alpha+1)}\left[\phi_{\alpha}(t) \log (k / t) g(\omega, t)\right]_{0}^{\pi} \\
& -\frac{1}{\Gamma(\alpha+1)} \int_{0}^{\pi} d\left\{\phi_{\alpha}(t) \log (k / t)\right\} g(\omega, t) .
\end{aligned}
$$

Hence

$$
\begin{aligned}
\int_{0}^{\pi} \phi(t) E(\omega, t) d t= & {\left[\sum_{1}^{\alpha}(-1)^{\rho-1} \Phi_{\rho}(t) E^{(\rho-1)}(\omega, t)\right]_{0}^{\pi} } \\
& +\frac{(-1)^{\alpha}}{\Gamma(\alpha+1)} \phi_{\alpha}(\pi) \log (k / \pi) g(\omega, \pi) \\
& +\frac{(-1)^{\alpha+1}}{\Gamma(\alpha+1)} \int_{0}^{\pi} d\left\{\phi_{\alpha}(t) \log (k / t)\right\} g(\omega, t) .
\end{aligned}
$$

Now $\Phi_{1}(\pi)=0$ by (1.22), $E^{(r)}(\omega, \pi)=0$, whenever $r$ is odd, $\phi_{\alpha}(\pi) \log (k / \pi)$ is a finite constant, and the integral

$$
\int_{0}^{\pi}\left|d\left\{\phi_{\alpha}(t) \log (k / t)\right\}\right|
$$

is convergent owing to the bounded variation of $\phi_{\alpha}(t) \log (k / t)$ in $(0, \pi)$. Hence it will suffice for the proof of the theorem to show that

$$
\int_{1}^{\infty} \frac{(\log \omega)^{1 / \alpha}}{\omega e^{\alpha+1}(\omega)}\left|E^{(\rho)}(\omega, \pi)\right| d \omega<\infty,
$$

where $\rho$ is an even integer such that $2 \leqq \rho \leqq \alpha-1$, 
(ii)

$$
\begin{aligned}
& \int_{1}^{\infty} \frac{(\log \omega)^{1 / \alpha}}{\omega e^{\alpha+1}(\omega)}|g(\omega, \pi)| d \omega<\infty, \\
& \int_{1}^{\infty} \frac{(\log \omega)^{1 / \alpha}}{\omega e^{\alpha+1}(\omega)}|g(\omega, t)| d \omega=O(1) \quad \text { for } 0<t<\pi .
\end{aligned}
$$

The result (i) has been established in Lemma 6. Again

$$
g(\omega, t)=g(\omega, \pi)-h(\omega, t) .
$$

Therefore

$$
\begin{aligned}
& \int_{1}^{\infty} \frac{(\log \omega)^{1 / \alpha}}{\omega e^{\alpha+1}(\omega)}|g(\omega, t)| d \omega=\left(\int_{1}^{\tau}+\int_{\tau}^{\infty}\right) \frac{(\log \omega)^{1 / \alpha}}{\omega e^{\alpha+1}(\omega)}|g(\omega, t)| d \omega \\
&\left(\text { where } \tau=(k / t)\{\log (k / t)\}^{1 / \alpha}\right) \\
& \leqq \int_{1}^{\tau} \frac{(\log \omega)^{1 / \alpha}}{\omega e^{\alpha+1}(\omega)}|g(\omega, t)| d \omega \\
&+\int_{1}^{\infty} \frac{(\log \omega)^{1 / \alpha}}{\omega e^{\alpha+1}(\omega)}|g(\omega, \pi)| d \omega \\
&+\int_{\tau}^{\infty} \frac{(\log \omega)^{1 / \alpha}}{\omega e^{\alpha+1}(\omega)}|h(\omega, t)| d \omega,
\end{aligned}
$$

since

$$
\int_{\tau}^{\infty} \frac{(\log \omega)^{1 / \alpha}}{\omega e^{\alpha+1}(\omega)}|g(\omega, \pi)| d \omega \leqq \int_{1}^{\infty} \frac{(\log \omega)^{1 / \alpha}}{\omega e^{\alpha+1}(\omega)}|g(\omega, \pi)| d \omega .
$$

Hence, Theorem 1 will be established if only the following are proved.

$$
\begin{array}{ll}
I_{1} & =\int_{1}^{\infty} \frac{(\log \omega)^{1 / \alpha}}{\omega e^{\alpha+1}(\omega)}|g(\omega, \pi)| d \omega<\infty ; \\
I_{2} & =\int_{1}^{\tau} \frac{(\log \omega)^{1 / \alpha}}{\omega e^{\alpha+1}(\omega)}|g(\omega, t)| d \omega=O(1) \quad \text { for } 0<t<\pi ; \\
I_{3} & =\int_{\tau}^{\infty} \frac{(\log \omega)^{1 / \alpha}}{\omega e^{\alpha+1}(\omega)}|h(\omega, t)| d \omega=O(1) \quad \text { for } 0<t<\pi .
\end{array}
$$

Proof of (3.11). Since

$$
\begin{aligned}
E^{(\alpha)}(\omega, u) & =\sum_{n \leqq \omega}\{e(\omega)-e(n)\}^{\alpha} e(n)\left(\frac{d}{d u}\right)^{\alpha} \cos n u, \\
|g(\omega, \pi)| & =\left|\sum_{n \leqq \omega}\{e(\omega)-e(n)\}^{\alpha} e(n) n^{\alpha} \int_{0}^{\pi} \frac{u^{\alpha}}{\log (k / u)} \cos n u d u\right|,
\end{aligned}
$$


or

$$
\left|\sum_{n \leqq \omega}\{e(\omega)-e(n)\}^{\alpha} e(n) n^{\alpha} \int_{0}^{\pi} \frac{u^{\alpha}}{\log (k / u)} \sin n u d u\right|,
$$

according as $\alpha$ is even or odd. Thus proving the convergence of the integral $I_{1}$ is the same thing as proving the summability $|R, e(\omega), \alpha+1|$ of the series $\sum n^{\alpha} \lambda_{n}$, where $\lambda_{n}$ is the Fourier cosine-constant of the even function $u^{\alpha} / \log |k / u|$, defined by periodicity outside $(-\pi, \pi)$, or of the series $\sum n^{\alpha} \mu_{n}$, where $\mu_{n}$ is the Fourier sine-constant of the odd function $u^{\alpha} / \log |k / u|$, defined by periodicity outside $(-\pi, \pi)$, according as $\alpha$ is even or odd.

Let $\alpha$ be even, and let

$$
\left(\frac{d}{d u}\right)^{\alpha}\left\{u^{\alpha} / \log \left|\frac{k}{u}\right|\right\} \sim \frac{1}{2} A_{\alpha}+\sum \epsilon_{n} \cos n u .
$$

Integrating successively, we have

$$
\begin{gathered}
u^{\alpha} / \log \left|\frac{k}{u}\right|-\frac{1}{2}\left(\frac{A_{\alpha} u^{\alpha}}{\alpha !}+\frac{A_{\alpha-2} u^{\alpha-2}}{(\alpha-2) !}+\cdots+A_{0}\right) \\
\sim \sum(-1)^{\alpha / 2} \frac{\epsilon_{n} \cos n u}{n^{\alpha}} .
\end{gathered}
$$

Let

$$
\frac{A_{\alpha} u^{\alpha}}{\alpha !}+\frac{A_{\alpha-2} u^{\alpha-2}}{(\alpha-2) !}+\cdots+A_{0} \sim \sum 2 \lambda_{n}^{\prime} \cos n u .
$$

Then, since $u^{\alpha} / \log |k / u| \sim \sum \lambda_{n} \cos n u$, (3.15) yields

$$
\sum n^{\alpha} \lambda_{n}=(-1)^{\alpha / 2} \sum \epsilon_{n}+\sum n^{\alpha} \lambda_{n}^{\prime} .
$$

Now, from (3.14),

$$
\begin{aligned}
c_{1} / \log \left|\frac{k}{u}\right|+c_{2} /\left(\log \left|\frac{k}{u}\right|\right)^{2}+\cdots+c_{\alpha+1} /(\log \mid & \left.\left|\frac{k}{u}\right|\right)^{\alpha+1} \\
& \sim \frac{1}{2} A_{\alpha}+\sum \epsilon_{n} \cos n u,
\end{aligned}
$$

where

$$
\begin{aligned}
\left(\frac{d}{d u}\right)^{\alpha}\left\{u^{\alpha} / \log (k / u)\right\}=c_{1} / \log \left(\frac{k}{u}\right)+c_{2} /\left(\log \frac{k}{u}\right)^{2} & +\cdots \\
& +c_{\alpha+1} /\left(\log \frac{k}{u}\right)^{\alpha+1} .
\end{aligned}
$$


If, now,

$$
\begin{aligned}
& c_{1} / \log \left|\frac{k}{u}\right| \sim \sum \delta_{1, n} \cos n u, \\
& c_{2} /\left(\log \left|\frac{k}{u}\right|\right)^{2} \sim \sum \delta_{2, n} \cos n u, \\
& \ldots \ldots . . . . . . . . . . \\
& c_{\alpha+1} /\left(\log \left|\frac{k}{u}\right|\right)^{\alpha+1} \sim \sum \delta_{\alpha+1, n} \cos n u,
\end{aligned}
$$

then, since $\sum_{n}\left|\delta_{v, n}\right|$ is convergent for $\nu=1,2, \cdots, \alpha+1$, by Lemma 2, we readily conclude that $\sum \epsilon_{n}$ is absolutely convergent, and hence a fortiori summable $|R, e(\omega), \alpha+1|$.

Also, if $\alpha=2 m$,

$$
\lambda_{n}^{\prime}=\pi^{-1} \sum_{1}^{m} \frac{A_{2 \mu}}{(2 \mu) !} \int_{0}^{\pi} u^{2 \mu} \cos n u d u
$$

But

$$
\int_{0}^{\pi} u^{2 \mu} \cos n u d u=(-1)^{n} \sum_{1}^{\mu}(-1)^{\rho-1} \frac{(2 \mu) !}{(2 \mu-2 \rho+1) !} \pi^{2 \mu-2 \rho+1} n^{-2 \rho}
$$

so that

$$
n^{\alpha} \lambda_{n}^{\prime}=\pi^{-1}(-1)^{n} \sum_{1}^{m}(-1)^{\rho-1} n^{2 m-2 \rho} \sum_{\rho}^{m} \frac{A_{2 \mu} \pi^{2 \mu-2 \rho+1}}{(2 \mu-2 \rho+1) !} .
$$

Therefore, by Lemma $11, \sum n^{\alpha} \lambda_{n}^{\prime}$ is summable $|R, e(\omega), \alpha+1|$. Hence from (3.16) it follows that $\sum n^{\alpha} \lambda_{n}$ is summable $|R, e(\omega), \alpha+1|$.

The case in which $\alpha$ is odd can be treated similarly.

Proof of (3.12).

$$
\begin{array}{rlr}
g(\omega, t) & =\int_{0}^{t} \frac{u^{\alpha}}{\log (k / u)} E^{(\alpha)}(\omega, u) d u \\
& =\frac{t^{\alpha}}{\log (k / t)} \int_{\eta}^{t} \frac{\partial}{\partial u} E^{(\alpha-1)}(\omega, u) d u \quad(0<\eta<t) \\
& =O\left[\frac{t^{\alpha}}{\log (k / t)} \sum_{n \leqq \omega}\{e(\omega)-e(n)\}^{\alpha} e(n) n^{\alpha-1}\right] \\
& =O\left[\frac{t^{\alpha}}{\log (k / t)} \omega^{\alpha} e^{\alpha+1}(\omega) /(\log \omega)^{1 / \alpha}\right],
\end{array}
$$$$
\text { (by the second mean value theorem) }
$$ 
by Lemma 7. Therefore

$$
\begin{array}{rlr}
I_{2} & =O\left\{\int_{1}^{\tau} \frac{(\log \omega)^{1 / \alpha}}{\omega e^{\alpha+1}(\omega)} \frac{t^{\alpha}}{\log (k / t)} \frac{\omega^{\alpha}}{(\log \omega)^{1 / \alpha}} e^{\alpha+1}(\omega) d \omega\right\} \\
& =O\left(\frac{t^{\alpha}}{\log (k / t)} \int_{1}^{\tau} \omega^{\alpha-1} d \omega\right) & \text { for } 0<t<\pi .
\end{array}
$$

\section{Proof of (3.13).}

$$
h(\omega, t)=\int_{t}^{\pi} \frac{u^{\alpha}}{\log (k / u)} E^{(\alpha)}(\omega, u) d u
$$

$$
\begin{aligned}
= & {\left[\frac{u^{\alpha}}{\log (k / u)} E^{(\alpha-1)}(\omega, u)\right]_{t}^{\pi}-\int_{t}^{\pi} \frac{d}{d u}\left\{\frac{u^{\alpha}}{\log (k / u)}\right\} E^{(\alpha-1)}(\omega, u) d u } \\
= & \frac{\pi^{\alpha}}{\log (k / \pi)} E^{(\alpha-1)}(\omega, \pi)-\frac{t^{\alpha}}{\log (k / t)} E^{(\alpha-1)}(\omega, t) \\
& -\int_{t}^{\pi}\left[\frac{\alpha u^{\alpha-1}}{\log (k / u)}+\frac{u^{\alpha-1}}{\{\log (k / u)\}^{2}}\right] E^{(\alpha-1)}(\omega, u) d u
\end{aligned}
$$$$
=O\left\{\left|E^{(\alpha-1)}(\omega, \pi)\right|\right\}+O\left\{\frac{t^{\alpha}}{\log (k / t)}\left|E^{(\alpha-1)}(\omega, t)\right|\right\}
$$$$
+O\left\{\int_{t}^{\pi} \frac{u^{\alpha-1}}{\log (k / u)}\left|E^{(\alpha-1)}(\omega, u)\right| d u\right\}
$$$$
=O\left\{\left|E^{(\alpha-1)}(\omega, \pi)\right|\right\}+O\left\{\frac{1}{t \log (k / t)} \cdot \frac{\log \omega}{\omega} e^{\alpha+1}(\omega)\right\}
$$$$
+O\left\{\frac{\log \omega}{\omega} e^{\alpha+1}(\omega) \int_{t}^{\pi} \frac{d u}{u^{2} \log (k / u)}\right\}
$$

$$
=O\left\{\left|E^{(\alpha-1)}(\omega, \pi)\right|\right\}+O\left\{\frac{1}{t \log (k / t)} \frac{\log \omega}{\omega} e^{\alpha+1}(\omega)\right\},
$$

since $\int_{t}^{\pi} d u / u^{2} \log (k / u)=O(1 / t \log (k / t))$. Therefore, observing that $E^{(\alpha-1)}(\omega$, $\pi)=0$, when $\alpha$ is even, and employing Lemma 6 , when $\alpha$ is odd, we obtain

$$
\begin{array}{rlr}
I_{3} & =O(1)+O\left(\int_{\tau}^{\infty} \frac{(\log \omega)^{1 / \alpha}}{\omega e^{\alpha+1}(\omega)} \frac{1}{t \log (k / t)} \frac{\log \omega}{\omega} e^{\alpha+1}(\omega) d \omega\right) \\
& =O(1)+O\left(\frac{1}{t \log (k / t)} \int_{\tau}^{\infty} \frac{(\log \omega)^{1+1 / \alpha}}{\omega^{2}} d \omega\right) \\
& =O(1) & \text { for } 0<t<\pi .
\end{array}
$$


This completes the proof of Theorem 1.

\subsection{Proof of Theorem 2.} form.

In view of Lemma 13, Theorem 2 can be put in the following equivalent

TheOREM 2a. If $\alpha$ is an integer $\geqq 1$, and if (i) $\int_{0}^{\pi} \log (k / t)\left|d \psi_{\alpha}(t)\right|<\infty$ and (ii) $\psi_{\alpha}(+0)=0$, then the conjugate series of the Fourier series of $f(t)$, at $t=x$, is summable $|R, e(\omega), \alpha+1|$.

We proceed to prove Theorem 2a. Since

$$
B_{n}(x)=\frac{2}{\pi} \int_{0}^{\pi} \psi(t) \sin n t d t,
$$

we have only to show that, under the hypotheses of the theorem, the integral

$$
\int_{1}^{\infty} \frac{(\log \omega)^{1 / \alpha}}{\omega e^{\alpha+1}(\omega)}\left|\int_{0}^{\pi} \psi(t) \bar{E}(\omega, t) d t\right| d \omega
$$

is convergent. Integrating by parts $\alpha$ times,

$$
\begin{aligned}
\int_{0}^{\pi} \psi(t) \bar{E}(\omega, t) d t= & {\left[\sum_{1}^{\alpha}(-1)^{\rho-1} \Psi_{\rho}(t) \bar{E}^{(\rho-1)}(\omega, t)\right]_{0}^{\pi} } \\
& +(-1)^{\alpha} \int_{0}^{\pi} \Psi_{\alpha}(t) \bar{E}^{(\alpha)}(\omega, t) d t \\
= & {\left[\sum_{1}^{\alpha}(-1)^{\rho-1} \Psi_{\rho}(t) \bar{E}^{(\rho-1)}(\omega, t)\right]_{0}^{\pi} } \\
& +\frac{(-1)^{\alpha}}{\Gamma(\alpha+1)}\left[\psi_{\alpha}(t) \chi(t)\right]_{0}^{\pi} \\
& +\frac{(-1)^{\alpha+1}}{\Gamma(\alpha+1)} \int_{0}^{\pi} d \psi_{\alpha}(t) \chi(t)
\end{aligned}
$$

where

$$
\begin{aligned}
\chi(t)= & \int_{0}^{t} u^{\alpha} \bar{E}^{(\alpha)}(\omega, u) d u=t^{\alpha} \bar{E}^{(\alpha-1)}(\omega, t)-\alpha t^{\alpha-1} \bar{E}^{(\alpha-2)}(\omega, t) \\
& +\alpha(\alpha-1) t^{\alpha-2} \bar{E}^{(\alpha-3)}(\omega, t)+\cdots+(-1)^{\alpha-1} \alpha(\alpha-1) \cdots 2 t \bar{E}(\omega, t) \\
& +(-1)^{\alpha-1} \alpha(\alpha-1) \cdots 2 \sum_{n \leqq \omega}\{e(\omega)-e(n)\}^{\alpha} e(n)\left(\frac{\cos n t}{n}-\frac{1}{n}\right) \\
= & \Lambda(t)+(-1)^{\alpha-1} \alpha(\alpha-1) \cdots 2 \sum_{n \leqq \omega}\{e(\omega)-e(n)\}^{\alpha} e(n)\left(\frac{\cos n t}{n}-\frac{1}{n}\right), \text { say, }
\end{aligned}
$$

Hence, it will suffice for the proof of the theorem to show that 


$$
\int_{1}^{\infty} \frac{(\log \omega)^{1 / \alpha}}{\omega e^{\alpha+1}(\omega)}\left|\bar{E}^{(\rho)}(\omega, \pi)\right| d \omega<\infty,
$$

where $\rho$ is an odd integer such that $1 \leqq \rho \leqq \alpha-1$,

$$
\begin{gathered}
J=\int_{1}^{\infty} \frac{(\log \omega)^{1 / \alpha}}{\omega e^{\alpha+1}(\omega)}\left|\int_{0}^{\pi} d \psi_{\alpha}(t) \Lambda(t)\right| d \omega<\infty \\
\int_{1}^{\infty} \frac{(\log \omega)^{1 / \alpha}}{\omega e^{\alpha+1}(\omega)} \mid \sum_{n \geqq \omega}\{e(\omega)-e(n)\}^{\alpha} e(n)\left\{-\psi_{\alpha}(\pi) \frac{\cos n \pi}{n}\right. \\
\left.+\int_{0}^{\pi} \frac{\cos n t}{n} d \psi_{\alpha}\right\} \mid d \omega<\infty .
\end{gathered}
$$

Proof of (3.21). The result has been established in Lemma 10 .

Proof of (3.22).

$$
\begin{aligned}
J & \leqq \int_{1}^{\infty} \frac{(\log \omega)^{1 / \alpha}}{\omega e^{\alpha+1}(\omega)}\left\{\int_{0}^{\pi}\left|d \psi_{\alpha}(t)\right||\Lambda(t)|\right\} d \omega \\
& =\left(\int_{1}^{\tau}+\int_{\tau}^{\infty}\right) \frac{(\log \omega)^{1 / \alpha}}{\omega e^{\alpha+1}(\omega)}\left\{\int_{0}^{\pi}\left|d \psi_{\alpha}(t)\right||\Lambda(t)|\right\} d \omega \\
& \left.\quad \quad \text { (where } \tau=(k / t)\{\log (k / t)\}^{1 / \alpha}\right) \\
& =J_{1}+J_{2}, \text { say. }
\end{aligned}
$$

Now, for the proof of the convergence of $J_{1}$ it is sufficient to show that

$$
\int_{0}^{\pi}\left|d \psi_{\alpha}(t)\right| \int_{1}^{\tau} \frac{(\log \omega)^{1 / \alpha}}{\omega e^{\alpha+1}(\omega)} t^{\rho+1}\left|\bar{E}^{(\rho)}(\omega, t)\right| d \omega<\infty,
$$

where $\rho$ is zero or a positive integer $\leqq \alpha-1$. Now

$$
\begin{aligned}
& \int_{0}^{\pi}\left|d \psi_{\alpha}(t)\right| \int_{1}^{r} \frac{(\log \omega)^{1 / \alpha}}{\omega e^{\alpha+1}(\omega)} t^{\rho+1}\left|\bar{E}^{(\rho)}(\omega, t)\right| d \omega \\
& =O\left\{\int_{0}^{\pi}\left|d \psi_{\alpha}(t)\right| \int_{1}^{r} \frac{(\log \omega)^{1 / \alpha}}{\omega e^{\alpha+1}(\omega)} t^{\rho+1}\left(\sum_{n \leqq \omega}\{e(\omega)-e(n)\}^{\alpha} e(n) n^{\rho}\right) d \omega\right\} \\
& =O\left\{\int_{0}^{\pi} \log (k / t)\left|d \psi_{\alpha}(t)\right| \frac{t^{\rho+1}}{\log (k / t)} \int_{1}^{r} \omega^{\rho} d \omega\right\} \quad \text { (by Lemma } \\
& =O(1),
\end{aligned}
$$

since $\left(t^{p+1} / \log (k / t)\right) \int_{1}^{\tau} \omega^{\rho} d \omega=O(1)$ for $0<t<\pi$.

To prove the convergence of $J_{2}$ we observe that

$$
\Lambda(t)=O\left\{(\log \omega / \omega) e^{\alpha+1}(\omega) t^{-1}\right\},
$$

by virtue of Lemma 9 . Hence 


$$
\begin{aligned}
J_{2} & =\int_{\tau}^{\infty} \frac{(\log \omega)^{1 / \alpha}}{\omega e^{\alpha+1}(\omega)}\left\{\int_{0}^{\pi}\left|d \psi_{\alpha}(t)\right||\Lambda(t)|\right\} d \omega \\
& =O\left\{\int_{0}^{\pi}\left|d \psi_{\alpha}(t)\right| \int_{\tau}^{\infty} \frac{(\log \omega)^{1 / \alpha}}{\omega e^{\alpha+1}(\omega)} \frac{\log \omega}{\omega} e^{\alpha+1}(\omega) t^{-1} d \omega\right\} \\
& =O\left\{\int_{0}^{\pi} \log (k / t)\left|d \psi_{\alpha}(t)\right|\{t \log (k / t)\}^{-1} \int_{\tau}^{\infty} \frac{(\log \omega)^{1+1 / \alpha}}{\omega^{2}} d \omega\right\} \\
& =O(1),
\end{aligned}
$$

since

$$
\{t \log (k / t)\}^{-1} \int_{\tau}^{\infty} \frac{(\log \omega)^{1+1 / \alpha}}{\omega^{2}} d \omega=O(1) \quad \text { for } 0<t<\pi
$$

Proof of (3.23). Proving (3.23) is the same thing as proving that the series $\sum u_{n}$, where

$$
u_{n}=-\psi_{\alpha}(\pi) \frac{\cos n \pi}{n}+\int_{0}^{\pi} \frac{\cos n t}{n} d \psi_{\alpha}(t)
$$

is summable $|R, e(\omega), \alpha+1|$. By Lemma 14 we conclude that $\sum u_{n}$ is summable $\left|R, \exp \left(\omega^{\delta}\right), 1\right|(0<\delta<1)$, and therefore by Lemmas 12 and 1 it is summable $|R, e(\omega), \alpha+1|$.

This completes the proof of Theorem 2 .

3.3. Proof of Theorem 3. Let $r$ be even. Then we have to show that, under the hypotheses of the theorem, the integral

$$
\int_{1}^{\infty} \frac{(\log \omega)^{1 / \alpha}}{\omega e^{\alpha+1}(\omega)}\left|\int_{0}^{\pi} \phi(t) E^{(r)}(\omega, t) d t\right| d \omega
$$

is convergent. Now

$$
\begin{aligned}
\int_{0}^{\pi} \phi(t) E^{(r)}(\omega, t) d t= & \frac{1}{2} \int_{0}^{\pi}\{P(t)+P(-t)\} E^{(r)}(\omega, t) d t \\
& +\int_{0}^{\pi} g(t) E^{(r)}(\omega, t) d t .
\end{aligned}
$$

Thus it is sufficient for our purpose to show that

$$
\int_{1}^{\infty} \frac{(\log \omega)^{1 / \alpha}}{\omega e^{\alpha+1}(\omega)}\left|\int_{0}^{\pi} \frac{1}{2}\{P(t)+P(-t)\} E^{(r)}(\omega, t) d t\right| d \omega<\infty,
$$




$$
\int_{1}^{\infty} \frac{(\log \omega)^{1 / \alpha}}{\omega e^{\alpha+1}(\omega)}\left|\int_{0}^{\pi} g(t) E^{(r)}(\omega, t) d t\right| d \omega<\infty .
$$

Proving (3.31) is the same thing as proving the summability $\mid R, e(\omega)$, $\alpha+1 \mid$ of $\sum n^{r} p_{n}$, where $p_{n}$ is the Fourier cosine-constant of the even function $\{P(t)+P(-t)\} / 2$. This can be easily proved by making use of Lemma 11 as in the proof of (3.11).

Next, to prove (3.32), if $\alpha>r$, integrating $\alpha-r$ times by parts,

$$
\begin{aligned}
\int_{0}^{\pi} g(t) E^{(r)}(\omega, t) d t= & {\left[\sum_{1}^{\alpha-r}(-1)^{\rho-1} G_{\rho}(t) E^{(r+\rho-1)}(\omega, t)\right]_{0}^{\pi} } \\
& +(-1)^{\alpha-r} \int_{0}^{\pi} G_{\alpha-r}(t) E^{(\alpha)}(\omega, t) d t .
\end{aligned}
$$

Now

$$
\begin{aligned}
\int_{0}^{\pi} G_{\alpha-r}(t) E^{(\alpha)}(\omega, t) d t= & \frac{1}{\Gamma(\alpha-r+1)} \int_{0}^{\pi} \gamma_{\alpha, r}(t) \log (k / t) \frac{t^{\alpha}}{\log (k / t)} E^{(\alpha)}(\omega, t) d t \\
= & \frac{1}{\Gamma(\alpha-r+1)}\left[\gamma_{\alpha, r}(t) \log (k / t) g(\omega, t)\right]_{0}^{\pi} \\
& -\frac{1}{\Gamma(\alpha-r+1)} \int_{0}^{\pi} d\left\{\gamma_{\alpha, r}(t) \log (k / t)\right\} g(\omega, t) .
\end{aligned}
$$

Hence, if $\alpha>r$,

$$
\begin{aligned}
\int_{0}^{\pi} g(t) E^{(r)}(\omega, t) d t= & {\left[\sum_{1}^{\alpha-r}(-1)^{\rho-1} G_{\rho}(t) E^{(r+\rho-1)}(\omega, t)\right]_{0}^{\pi} } \\
& +\frac{(-1)^{\alpha-r}}{\Gamma(\alpha-r+1)} \gamma_{\alpha, r}(\pi) \log (k / \pi) g(\omega, \pi) \\
& +\frac{(-1)^{\alpha-r+1}}{\Gamma(\alpha-r+1)} \int_{0}^{\pi} d\left\{\gamma_{\alpha, r}(t) \log (k / t)\right\} g(\omega, t) .
\end{aligned}
$$

Also, if $\alpha=r$,

$$
\int_{0}^{\pi} g(t) E^{(r)}(\omega, t) d t=\gamma_{r, r}(\pi) \log (k / \pi) g(\omega, \pi)-\int_{0}^{\pi} d\left\{\gamma_{r, r}(t) \log (k / t)\right\} g(\omega, t) .
$$


Hence, as in the proof of Theorem 1, it is sufficient for our purpose to establish only the following.

$$
\begin{aligned}
& \int_{1}^{\infty} \frac{(\log \omega)^{1 / \alpha}}{\omega e^{\alpha+1}(\omega)}|g(\omega, \pi)| d \omega<\infty \\
& \int_{1}^{\tau} \frac{(\log \omega)^{1 / \alpha}}{\omega e^{\alpha+1}(\omega)}|g(\omega, t)| d \omega=O(1) \quad \text { for } 0<t<\pi
\end{aligned}
$$

and

$$
\int_{\tau}^{\infty} \frac{(\log \omega)^{1 / \alpha}}{\omega e^{\alpha+1}(\omega)}|h(\omega, t)| d \omega=O(1) \quad \text { for } 0<t<\pi
$$

All these results have been proved in $\$ 3.1$.

The case in which $r$ is odd can be dealt with similarly. This completes the proof of Theorem 3.

3.4. Proof of Thorem 4. Let $r$ be even. Then we have to show that, under the hypotheses of the theorem, the integral

$$
\int_{1}^{\infty} \frac{(\log \omega)^{1 / \alpha}}{\omega e^{\alpha+1}(\omega)}\left|\int_{0}^{\pi} \psi(t) \bar{E}^{(r)}(\omega, t) d t\right| d \omega
$$

is convergent. Now

$$
\begin{aligned}
\int_{0}^{\pi} \psi(t) \bar{E}^{(r)}(\omega, t) d t= & \int_{0}^{\pi} \frac{1}{2}\{P(t)-P(-t)\} \bar{E}^{(r)}(\omega, t) d t \\
& +\int_{0}^{\pi} h(t) \bar{E}^{(r)}(\omega, t) d t .
\end{aligned}
$$

Thus it is sufficient for our purpose to show that

$$
\begin{gathered}
\int_{1}^{\infty} \frac{(\log \omega)^{1 / \alpha}}{\omega e^{\alpha+1}(\omega)}\left|\int_{0}^{\pi} \frac{1}{2}\{P(t)-P(-t)\} \bar{E}^{(r)}(\omega, t) d t\right| d \omega<\infty, \\
\int_{1}^{\infty} \frac{(\log \omega)^{1 / \alpha}}{\omega e^{\alpha+1}(\omega)}\left|\int_{0}^{\pi} h(t) \bar{E}^{(r)}(\omega, t) d t\right| d \omega<\infty .
\end{gathered}
$$

Proving (3.41) is the same thing as proving the summability $\mid R, e(\omega)$, $\alpha+1 \mid$ of $\sum n^{r} q_{n}$, where $q_{n}$ is the Fourier sine-constant of the odd function $\{P(t)-P(-t)\} / 2$. This can be easily proved like (3.31).

Next, to prove (3.42), if $\alpha>r$, integrating $\alpha-r$ times by parts, 


$$
\begin{aligned}
\int_{0}^{\pi} h(t) \bar{E}^{(r)}(\omega, t) d t= & {\left[\sum_{1}^{\alpha-r}(-1)^{\rho-1} H_{\rho}(t) \bar{E}^{(r+\rho-1)}(\omega, t)\right]_{0}^{\pi} } \\
& +(-1)^{\alpha-r} \int_{0}^{\pi} H_{\alpha-r}(t) \bar{E}^{(\alpha)}(\omega, t) d t \\
= & {\left[\sum_{1}^{\alpha-r}(-1)^{\rho-1} H_{\rho}(t) \bar{E}^{(r+\rho-1)}(\omega, t)\right]_{0}^{\pi} } \\
& +\frac{(-1)^{\alpha-r}}{\Gamma(\alpha-r+1)}\left[\theta_{\alpha, r}(t) \chi(t)\right]_{0}^{\pi} \\
& +\frac{(-1)^{\alpha-r+1}}{\Gamma(\alpha-r+1)} \int_{0}^{\pi} d \theta_{\alpha, r}(t) \chi(t),
\end{aligned}
$$

where $\chi(t)$ has the same meaning as in $\$ 3.2$.

Also, if $\alpha=r$,

$$
\int_{0}^{\pi} h(t) \bar{E}^{(r)}(\omega, t) d t=\left[\theta_{r, r}(t) \chi(t)\right]_{0}^{\pi}-\int_{0}^{\pi} d \theta_{r, r}(t) \chi(t)
$$

Hence, as in the proof of Theorem 2, it is sufficient for our purpose to establish only the following.

$$
\int_{1}^{\infty} \frac{(\log \omega)^{1 / \alpha}}{\omega e^{\alpha+1}(\omega)}\left|\bar{E}^{(\rho)}(\omega, \pi)\right| d \omega<\infty,
$$

where $\rho$ is an odd integer such that $1 \leqq \rho \leqq \alpha-1$,

$$
\int_{1}^{\infty} \frac{(\log \omega)^{1 / \alpha}}{\omega e^{\alpha+1}(\omega)}\left|\int_{0}^{\pi} d \theta_{\alpha, r}(t) \Lambda(t)\right| d \omega<\infty
$$

and

$$
\begin{aligned}
\int_{1}^{\infty} \frac{(\log \omega)^{1 / \alpha}}{\omega e^{\alpha+1}(\omega)} \mid \sum_{n \leqq \omega}\{e(\omega)-e(n)\}^{\alpha} e(n) & \left\{-\theta_{\alpha, r}(\pi) \frac{\cos n \pi}{n}\right. \\
+ & \left.\int_{0}^{\pi} \frac{\cos n t}{n} d \theta_{\alpha, r}(t)\right\} \mid d \omega<\infty .
\end{aligned}
$$

In the arguments used in the proof of Theorem 2 we have only to replace $\psi_{\alpha}(t)$ by $\theta_{\alpha, r}(t)$ to establish the above results.

The case in which $r$ is odd can be dealt with similarly. This completes the proof of Theorem 4 . 


\section{BiBLIOGRAPHY}

1. K. Chandrasekharan, The second theorem of consistency for absolutely summable series, J. Indian Math. Soc. N.S. vol. 6 (1942) pp. 168-180.

2. G. H. Hardy, Divergent series, Oxford, 1949.

3. G. H. Hardy and M. Riesz, The general theory of Dirichlet series, Cambridge Tracts in Mathematics and Mathematical Physics, No. 18, 1915.

4. R. Mohanty, A criterion for the absolute convergence of a Fourier series, Proc. London Math. Soc. (2) vol. 51 (1949) pp. 186-196.

5. - On the absolute Riesz summability of Fourier series and allied series, Proc. London Math. Soc. (2) vol. 52 (1951) pp. 295-320.

6. N. Obrechkoff, Sur la sommation absolue des séries de Dirichlet, C.R. Acad. Sci. Paris vol. 186 (1928) pp. 215-217.

7. —, Über die absolute Summierung der Dirichletschen Reihen, Math. Zeit. vol 30 (1929) pp. 375-386.

8. C. de la Vallee Poussin, Course d'analyse infinitésimale, Louvain-Paris, 1923.

9. F. T. Wang, On Riesz summability of Fourier series: III, Proc. London Math. Soc. (2) vol. 51 (1950) pp. 215-231.

University of Allahabad,

Allahabad, India. 